\title{
A generalized eigenvalue distribution
}

\author{
Michael Handelman \\ Department of Physics, University of Michigan, Ann Arbor, Michigan 48109 \\ (Received 17 January 1978) \\ The statistical properties of the eigenvalues of random unitary matrices may be determined from the joint \\ probability density function of the matrix eigenvalues. Earlier theorems have derived the density function \\ for the unitary and symplectic circular ensembles from that for the circular orthogonal ensemble. A \\ method is presented here for successively eliminating variables from the probability density function for \\ the orthogonal circular ensemble; the method generalizes an earlier result, and the resulting function \\ appears to represent the behavior of eigenvalues from a new series of matrix ensembles.
}

The statistical properties of the eigenvalues of random unitary matrices, which have been studied by Dyson and Mehta, ${ }^{1}$ may be determined from the joint probability density function of the matrix eigenvalues. Such functions typically take the form

$$
P_{N^{\prime} B}\left(\theta_{1}, \cdots, \theta_{N}\right)=C_{N^{\prime} B} \prod_{j<k}\left|\exp \left(i \theta_{j}\right)-\exp \left(i \theta_{k}\right)\right|^{\beta},
$$

whe re $\beta=1,2$, or 4 , corresponding to matrices of the orthogonal, unitary or symplectic circular ensembles.

The motivation for this discussion is the knowledge that there are simple ways to transform the orthogonal circular ensemble eigenvalue density into the circular unitary or symplectic densities. These methods involve eliminating eigenvalues, according to a particular procedure, from the orthogonal ensemble. Such procedures can in principle be generalized, and this suggests the possibility that there is some significance to be attached to the density functions their application would determine. In what follows, we generate a class of functions by generalizing one such procedure.

\section{RELATIONS BETWEEN EIGENVALUE DENSITY FUNCTIONS}

The transformations referred to are two physically meaningful and nontrivial methods of generating the unitary and symplectic circular ensembles from the orthogonal ensemble.

That is, one can perform operations $D, G$ on the eigenvalues of the orthogonal circular ensemble such that

$D: P_{2 N^{\prime} 1}\left(\theta_{1}, \cdots, \theta_{2 N}\right) \rightarrow P_{N^{\prime} 4}\left(\theta_{2}, \theta_{4}, \cdots, \theta_{2 N}\right)$,

$G: \begin{aligned} & \text { Two copies of } \\ & P_{N^{\prime} 1}\left(\theta_{1}, \cdots, \theta_{N}\right)\end{aligned}-P_{N / 2}\left(\phi_{1}, \phi_{2}, \cdots, \phi_{N}\right)$.

These two transformations are defined by two theorems, the former due to Mehta and Dyson, ${ }^{2}$ the latter suggested by Dyson ${ }^{3}$ and proved by Gunson. ${ }^{4}$

The meaning of (2a) is that, given a sequence of $2 \mathrm{~N}$ eigenvalues belonging to the circular orthogonal ensemble, one may pick $N$ alternate eigenvalues from that sequence; the $N$ eigenvalues chosen will have the same statistical properties as those of a naturally occurring sequence of $N$ eigenvalues belonging to the symplectic ensemble. That is, the eigenvalue density functions of the former sequence (made by choosing alternate eigenvalues) and of the latter sequence (be longing to the symplectic ensemble) are of the same form. The theorem is stated explicitly below [Eq. (7)].

The relationship (2b) means that if one takes two independent sequences of eigenvalues belonging to the orthogonal ensemble, superimposes them (randomly), and then picks from that mixed sequence $N$ alternate eigenvalues, the chosen eigenvalues will have a density function which is the same as that for a unitary ensemble of order $N$.

There are thus explicit relations between $P_{2 N^{\prime} 1}$ and $P_{N^{\prime} 4}$, and between $P_{N^{\prime 1}}$ and $P_{N 2}$. Two obvious questions may be asked: Can we find a similar relationship between $P_{N^{\prime} 2}$ and $P_{N^{\prime} 4}$, and can either of the two relationships in Eq. (2) be generalized, for $\beta$ not restricted to the values $1,2,4$.

Both questions are reasonable, the first because we would like to know whether the three ensembles are symmetrical in their relationships to each other, the second on the supposition that generalized eigenvalue densities may have some statistical significance. Since the functions $P_{N^{\prime} \beta}$ as given by Dyson are well defined and properly normalized for all (complex) values of $\beta$-and intuitively appealing generalizations of the Mehta-Dyson or Gunson theorems should involve relationships between various $P_{N \cdot \beta}$ with positive integral $\beta$-the proposed generalizations should have at least a mathematical interest.

In answer to the questions posed above, we may say the following.

First, we have been unable to find a relationship between $P_{N^{\prime}}$ and $P_{N^{\prime} 4}$ with either physical or mathematical interest. This is not to say that such a relationship does not exist, but we are inclined to be doubtful. In view of the ground fields underlying the various ensembles, we note that (2a) implies in a sense a mapping between the real field and the quaternion field; $(2 b)$ is, again in a sense, a mapping between the real field and the complex field. A relation of the sort desired would be a similar kind of mapping between the complex field and the field of real quaternions.

As regards a generalization of $(2 b)$, the proper result would be a theorem that could predict the properties of a sequence of eigenvalues formed by superimposing two 
or more independent sequences, and picking $N$ eigenvalues, according to a prescribed formula, from the mixed sequence. If the probability density function for the eigenvalues of the resultant sequence were similar to $P_{N^{\prime} \beta}$, for some integral $\beta$, that might be a result of physical interest. We have no reason to believe that some such formula might not exist, but there is not one available at the present time.

A generalization of the theorem indicated by Eq. (2a) has been proved, and is given by Eq. ( 8 ) below. It depends on the possibility of writing certain kinds of products as confluent alternant ${ }^{5}$ determinants.

With the exception of the above argument based on the ground fields underlying the respective ensembles, there exists, to our knowledge, no explanation for the existence of the theorems implied by Eqs. (2a), (2b). However, Porter ${ }^{6}$ has suggested the possibility of restating (2a), (2b) for the Gaussian ensembles; if such restatements are in fact possible, it would seem that these theorems must be connected in a very powerful way with the foundations of random matrix theory.

It is possible to state a number of theorems similar to (8). Such a theorem must eliminate a particular number $(\beta N-N)$ of arguments (eigenvalues) from $P_{\beta N^{\prime} 1}$, and must do so in a particular number of steps (integrations). An essential element of the procedure, however, is that the arguments-eigenvalues or dummy variablesbe maintained in a certain fixed relationship to each other.

What (8) does is perform a series of integrations over the eigenvalues to be eliminated. The limits of integration may be other eigenvalues (in the last set of integrations), or dummy variables (in the preceding sets of integrations).

The limits of integration in the earlier integrations may, however, be other eigenvalues, provided that they are chosen in a symmetrical way from among only those eigenvalues over which the number of integrations to be performed is exactly the same as is that for the eigenvalues for which they will serve as limits; if any other eigenvalues were to be chosen as limits of integration, the essential fixed relationship between the arguments would be disrupted.

We have chosen the most general possible statement of the theorem, using dummy variables as limits of integration, rather than choosing one of the many possible equivalent statements without dummy variables.

\section{GENERALIZATION OF THE MEHTA-DYSON THEOREM}

The normalization constant $C_{N^{\prime} \beta}$ appearing in Eq. (1), which is correct for all values of $\beta$, is given by ${ }^{7}$

$$
C_{N \cdot \beta}=\frac{1}{(2 \pi)^{N}} \frac{\left[\Gamma\left(1+\frac{1}{2} \beta\right)\right]^{N}}{\Gamma\left(1+\frac{1}{2} N \beta\right)}
$$

If we order the angles

$$
0 \leqslant \theta_{1} \leqslant \theta_{2} \leqslant \cdots \leqslant \theta_{N} \leqslant 2 \pi,
$$

we can use the identity

$$
\begin{aligned}
& \left|\exp \left(i \theta_{m}\right)-\exp \left(i \theta_{n}\right)\right|=i^{-1} \exp \left[-\frac{1}{2} i\left(\theta_{m}+\theta_{n}\right)\right] \\
& \times\left(\exp \left[i \theta_{m}\right]-\exp \left[i \theta_{n}\right]\right), \quad \theta_{n} \leqslant \theta_{m},
\end{aligned}
$$

to write

$$
\begin{aligned}
P_{N^{\prime} 1}\left(\theta_{1}, \cdots, \theta_{N}\right) & =C_{N^{\prime} 1}(i)^{-N(N-1) / 2} \operatorname{det}\left[\exp \left(i j \theta_{s}\right)\right], \\
j & =\frac{-(N-1)}{2}, \frac{-(N-3)}{2}, \cdots, \frac{+(N-1)}{2}, \\
s & =1,2, \cdots, N_{0}
\end{aligned}
$$

Theorem (2a), relating the orthogonal and symplectic ensembles, is derived using the determinantal form (6) of the orthogonal ensemble eigenvalue density. It is given by

$$
\begin{aligned}
& \int_{\theta_{2 N-1}}^{\theta_{2 N}} d \theta_{2 N-1} \cdots \int_{\theta_{2}}^{\theta_{4}} d \theta_{3} \int_{\theta_{2 N^{-2 \pi}}}^{\theta^{2}} d \theta_{1} P_{2 N^{\prime} 1}\left(\theta_{1}, \cdots, \theta_{2 N}\right) \\
& \quad=\frac{C_{2 N^{\prime} 1}}{C_{N^{\prime} 4}} 2\left(\frac{2^{2 N} N !}{(2 N) !}\right)^{2} P_{N^{\prime} 4}\left(\theta_{2}, \theta_{4}, \cdots, \theta_{2 N}\right),
\end{aligned}
$$

where we have neglected constant factors on the righthand side of (7) that result from the ordering (4) of the eigenvalues.

Note that in (7), integrals are performed over the odd numbered eigenvalues between the neighboring even numbered eigenvalues; the results would clearly be unchanged if instead we were to integrate over the even numbered eigenvalues between the neighboring odd numbered eigenvalues.

As has already been indicated, the meaning of the theorem is that $N$ alternate eigenvalues, taken from a series of $2 N$ belonging to the orthogonal ensemble, are distributed in the same way as $N$ eigenvalues taken from the symplectic ensemble of order $N$. An obvious question to ask is, what will happen if instead of choosing alternate eigenvalues, we choose instead every third eigenvalue, or every fourth.

The confluent alternant determinant indicates how the choices of eigenvalues (the integrations) should be made: While the symplectic ensemble generated by (7) corresponds to the simplest sort of confluent alternant, generalizations of (7) (for $\beta>2$ ) will generate ensembles corresponding to confluent alternants of higher degree.

We are of course concerned with (eigenvalue density) functions that are symmetric in all their arguments. In general, a confluent alternant determinant can describe (symmetric) functions of the form $P_{N^{\prime} A^{2}}$ (for $A$ positive integral); these are the kinds of functions that are produced by the theorem below.

Theorem: For $\beta N$ even, $\beta, N$ positive integers, $N \geqslant 2$, $\beta \geqslant 2$,

$$
\left[\int_{\theta_{N \beta-2}}^{\theta_{\beta}} d \theta_{\beta-1}\left(\prod_{\alpha=1}^{N \infty 1} \int_{\theta_{q \beta}}^{\theta_{(\alpha+1) \beta}} d \theta_{(q+1) \beta-1}\right)\right]
$$




$$
\begin{aligned}
& \times\left[\prod_{\alpha=1}^{\beta-2} \int_{\theta_{N \beta-2 \pi}}^{\theta_{\beta}} d \phi_{\alpha}^{\beta-2}\left(\prod_{\beta=1}^{-1} \int_{\theta_{\alpha \beta}}^{\theta_{(\alpha+1) \beta}} d \phi_{\alpha \beta+\alpha}^{\beta-2}\right)\right]
\end{aligned}
$$

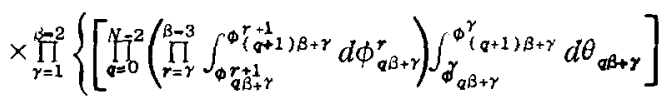

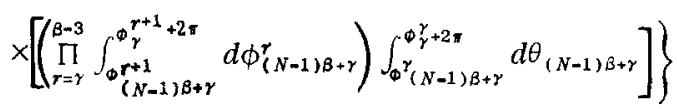

$$
\begin{aligned}
& \times P_{B N^{\prime} 1}\left(\theta_{1}, \ldots, \theta_{N \beta}\right) \\
& =\frac{1}{K}(i)^{N \beta(\beta-1) / 2} \frac{C_{\beta N^{\prime} 1}(i)^{-N \beta(N \beta-1) / 2}}{C_{N^{\prime} \beta}^{2}}[\Gamma(1) \cdot \cdots \Gamma(\beta)]^{N} \\
& \times(i)^{N \beta^{2}(N-1) / 2} 2^{(\beta-1)}( \pm 2)^{(\beta-1)(\beta-2) / 2} \quad / 2 \\
& \times P_{N^{\prime} \beta}^{2}\left(\theta_{\beta}, \theta_{2 \beta}, \ldots, \theta_{N \beta}\right) \text {. }
\end{aligned}
$$

The constant $K$ is given by

$$
K=\left[\prod_{p=1}^{\beta N}\left(i j_{p}\right)\right]^{\beta-1},
$$

where

$$
j_{1}=-\frac{(\beta N-1)}{2}, j_{2}=\frac{-(\beta N-3)}{2}, \ldots, j_{N}=\frac{+(\beta N-1)}{2},
$$

so that

$$
K=\left[(\beta N) ! /(2)^{\beta N}\left(\frac{\beta N}{2}\right) !\right]^{2(\beta-1)}
$$

We can then write the right-hand side of (8) as

$$
\begin{aligned}
= & {\left[\frac{2^{\beta N}(\beta N / 2) !}{(\beta N) !}\right]^{2(8-1)} \frac{C_{\beta N^{\prime}}}{C_{N^{\prime} \beta}^{2}}[\Gamma(1) \cdots \Gamma(\beta)]^{N_{2} \beta-1}( \pm 2)^{(\beta-1)(\beta-2) / 2} } \\
& \times P_{N^{\prime} \beta}{ }^{2}\left(\theta_{\beta}, \theta_{2 \beta}, \ldots, \theta_{N \beta}\right) .
\end{aligned}
$$

In (8) and (12) the + sign is for $N$ even, and the - sign is for $N$ odd.

Note that (8) reduces exactly to (7) in the case $\beta=2$ : the products over $\alpha, \gamma$, and $r$ all vanish; the remaining integrals are over $\theta_{p}, p$ odd, and have the correct limits. The constant, as can be seen in (12), also reduces to the proper form.

Note also the introduction of the $(\beta-2)$ series of dummy variables, $\phi_{p}^{\gamma}$, where $\gamma$ labels each series $(1 \leqslant \gamma \leqslant \beta-2)$; the subscript $p$ of the dummy variables will obey $\gamma \leqslant p \leqslant q \beta+\gamma(q=0,1, \ldots, N-1)$.

Proof of Theorem (8): We can write $P_{N \cdot \beta}{ }^{2}\left(\theta_{\beta}, \theta_{2 \beta}, \ldots, \theta_{N \beta}\right)$ as a confluent alternant determinant

$$
\begin{aligned}
P_{N^{\prime} \beta}{ }^{2}= & \frac{C_{N^{\prime} \beta}{ }^{2}}{\left.(i)^{\beta^{2} N\left(N^{-1) / 2}[\Gamma(1)\right.} \cdots \Gamma(\beta)\right]^{N}} \\
& \times \operatorname{det}\left[\exp \left(i j \theta_{p}\right), \quad j \exp \left(i j \theta_{p}\right), \ldots, j^{\beta-1} \exp \left(i j \theta_{p}\right)\right],
\end{aligned}
$$

where the column index $p$ takes the values $\beta, 2 \beta, \ldots, N \beta$, and the row index $j$ takes the values indicated in $(10)$.

Similarly, we can order the eigenvalues, $0 \leqslant \theta_{1} \leqslant \theta_{2}$ $\leqslant \cdots \leqslant \theta_{N B} \leqslant 2 \pi$, and use (5) and (6) to write as a determinant
$P_{B N} \cdot 1\left(\theta_{1}, \ldots, \theta_{B N}\right)=\frac{C_{\beta N^{\prime} 1}}{(i)^{\beta N(B N-1) / 2}} \operatorname{det}\left[\exp \left(i j \theta_{s}\right)\right]$,

where $j$ is given by $(10)$, and $s=1,2, \ldots, \beta N$.

In the statement of the theorem we neglect constant factors on the right-hand side of (8) that result from the ordering of the eigenvalues leading to (14).

In order to transform the determinant in (14) into the determinant in (13), divide the $\beta N$ variables into groups, with $\beta$ variables in each group. Consider the first set of $\beta$ variables: $\theta_{1}, \theta_{2}, \ldots, \theta_{\beta}$. The first variable- $\theta_{1}$-will be integrated a total of $\beta-1$ times; $\theta_{2}$ will be integrated a total of $\beta-2$ times; the $t$ th variable $(t=1,2, \ldots, \beta)$ will be integrated $\beta-t$ times.

Consider again the $t$ th variable. The first $\beta-t-1$ integrations will transform $\theta_{t}$ into a succession of dummy variables $-\phi^{1}, \phi^{2}, \ldots, \phi^{\beta-t-1}$. The final, $\beta-t$, integration will transform $\phi^{\beta-t-1}$ into $\theta_{\beta}$. In general, the first $\beta$ variables of $(14)-\theta_{1}, \theta_{2}, \ldots, \theta_{\beta}$-are transformed finally into $\theta_{\beta}$. The next set of $\beta$ variables $\theta_{\beta+1}, \theta_{\beta+2}, \ldots, \theta_{2 \beta}$-is transformed finally into $\theta_{2 \beta}$. The final set of $\beta$ variables $-\theta_{(N-1) \beta+1}, \ldots, \theta_{N \beta}$-is transformed finally into $\theta_{N \beta}$.

The integrations in (8), inside the product over $\gamma$, encompass all the integrations that are necessary to transform any of the $\theta^{\prime}$ 's into the dummy variables $\phi^{1}$, and to transform any set of the dummy variables $\phi^{a}$ into the succeeding set of dummy variables $\phi^{a+1}$.

The other factors in (8) (those outside the product over $\gamma$ ) do the final integrations, by transforming the final sets of dummy variables into $\theta_{\beta}, \theta_{2 \beta}, \ldots, \theta_{N \beta}$. Of course, since there is only one integration to be performed on each of $\theta_{\beta-1}, \theta_{2 \beta-1}, \ldots, \theta_{N \beta-1}$, these variables are transformed directly into $\theta_{\beta}, \theta_{2 \beta}, \ldots, \theta_{N \beta}$, respectively。

The integrals in (8) are pertormed from right to left. Begin by considering the integrations inside the product over $\gamma$, which act on variables initially labeled $\theta_{\alpha \beta+\gamma}$, where $\gamma^{\prime}$ is a given, fixed value of $\gamma$, and $q=0,1, \ldots, N-2$. That is, consider

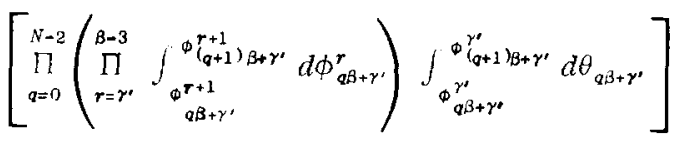

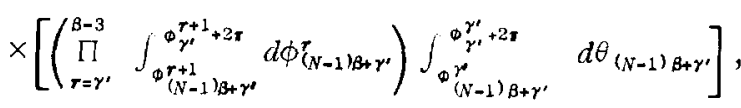

acting on $\operatorname{det}\left[\exp \left(i j \theta_{s}\right)\right]$.

These integrals do the first $\beta-t-1$ integrations on the $t$ th variable of each set of $\beta$ variables. The integrals in the second line of (15) do these integrations on the last set of $\beta$ variables $-\theta_{(N-1)_{3+1}}, \theta_{(N-1)_{\beta+2}}, \ldots, \theta_{N \beta}$-while the integrals in the first line of (15) do these integrations on all other sets of $\beta$ variables. Of course, as mentioned, the variables $\theta_{\beta}, \theta_{2 \beta}, \ldots, \theta_{N \beta}$ are not integrated over at all, while the variables $\theta_{\beta-1}, \theta_{2 \beta-1}, \ldots, \theta_{N \beta-1}$ are transformed directly into $\theta_{\beta}, \theta_{2 \beta}, \ldots, \theta_{N \beta}$ by integrals not included in (15) [by integrals in the first line of (8)]. 
Now, write out (15) explicitly for the fixed value $r=\gamma^{\prime}$. These integrals do not vanish for $\beta-3 \geqslant 1$, and are given by

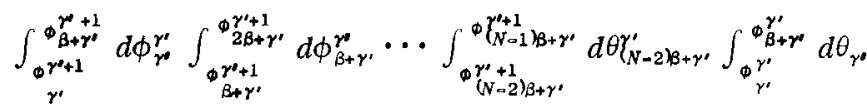

$$
\begin{aligned}
& \times \int_{\phi_{\beta+\gamma^{\prime}}^{\gamma^{*}}}^{\phi_{2 \beta+\gamma^{\prime}}^{\gamma^{\prime}}} d \theta_{\beta+\gamma^{\prime}} \cdots \int_{\phi_{(N-2) \beta+\gamma^{\prime}}^{\gamma^{\prime}}}^{\phi \gamma_{N-1) \beta+\gamma^{\prime}}^{\gamma^{\prime}}} d \theta_{(N-2) \beta+\gamma^{\prime}}
\end{aligned}
$$

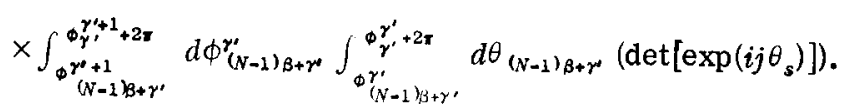

The integrations in (16) are performed only on the $\gamma^{\prime}$, $\beta+\gamma^{\prime}, 2 \beta+\gamma^{\prime}, \ldots,(N-1) \beta+\gamma^{\prime}$ columns of the matrix. The integrations over the $\theta$ 's transform these columns as shown: The columns

$$
\left\{\exp \left(i j \theta_{\gamma^{\prime}}\right), \exp \left(i j \theta_{\beta+\gamma^{\prime}}\right), \ldots, \exp \left[i j \theta_{(N-1)_{\beta+\gamma^{\prime}}}\right]\right\}
$$

become

$$
\begin{gathered}
\left\{\frac{\exp \left(i j \phi_{\beta^{\prime}+\gamma^{\prime}}^{\gamma^{\prime}}\right)-\exp \left(i j \phi_{\gamma^{\prime}}^{\gamma^{\prime}}\right)}{i j}, \frac{\exp \left(i j \phi_{2 \beta+\gamma^{\prime}}^{\gamma^{\prime}}\right)-\exp \left(i j \phi_{\beta+\gamma^{\prime}}^{\gamma^{\prime}}\right)}{i j},\right. \\
\left.\cdots, \frac{\exp \left[i j\left(\phi_{\gamma^{\prime}}^{\gamma^{\prime}}+2 \pi\right)\right]-\exp \left[i j \phi_{(N-1) \beta_{+\gamma^{\prime}}^{\gamma^{\prime}}}^{\gamma^{\prime}}\right]}{i j}\right\} 。
\end{gathered}
$$

Since $j$ is half-integral for $\beta N$ even, $\exp [i j(\phi+2 \pi)]$ $=-\exp (i j \phi)$, and we can add columns in (18) so as to obtain

$$
\pm\left\{\frac{\exp \left(i j \phi_{\gamma^{\prime}}^{\gamma^{\prime}}\right)}{i j}, \frac{\exp \left(i j \phi_{\beta_{+\gamma^{\prime}}}^{\gamma^{\prime}}\right)}{i j}, \ldots, \frac{\exp \left[i j \phi_{(N-1) \beta_{+} \gamma^{\prime}}^{\gamma^{\prime}}\right]}{i j}\right\},
$$

where the + sign holds for $N$ even, and the - sign for $N$ odd。

We can now perform the integrations over the $\phi^{\gamma /}$ 's, as indicated in (16), and the integrations over the $\phi^{\gamma^{\prime+1}}$, $\phi^{r+2}, \cdots$, until all of the $\beta-\gamma^{\prime}+1$ (with $t=\gamma^{\prime}$ ) integrations, over the $t, \beta+t, 2 \beta+t, \cdots$, columns have been performed.All the integrations proceed in essentially the same way as $(17)-(19)$; when the integrations in

(15) have all been performed, the $\gamma^{\prime}, \beta+\gamma^{\prime}, \cdots,(N-1) \beta+\gamma^{\prime}$ columns will have been transformed into

$( \pm 2)^{\beta-\gamma^{\prime}-1}\left\{\frac{\exp \left(i j \phi_{\gamma^{\prime}}^{\beta-2}\right)}{(i j)^{\beta-\gamma^{\prime}-1}}, \frac{\exp \left(i j \phi_{\beta^{\prime}+\gamma^{\prime}}^{\beta-2}\right)}{(i j)^{\beta-\gamma^{\prime}-1}}, \cdots, \frac{\exp \left[i j \phi_{(N-1) \beta+\gamma^{\prime}}^{\beta-2}\right.}{(i j)^{\beta-\gamma^{\prime}-1}}\right\}$

Since the integrals in (15) commute for different values of $\gamma^{\prime}$, it is clear that once they have been performed, in whatever order, we can perform all the integrals in (8) inside the product over $\gamma$, for $\gamma=1, \cdots$, $\beta-2$. These integrals transform

$$
\begin{aligned}
& \operatorname{det}\left[\exp \left(i j \theta_{s}\right)\right] \rightarrow( \pm 2)^{(\beta-1)(\beta-2) / 2} \operatorname{det}\left\{\frac{\exp \left(i j \phi_{\phi \beta+2}^{\beta-2}\right)}{(i j)^{\beta-2}},\right. \\
& \quad \frac{\exp \left(i j \phi_{\beta \beta+2}^{\beta-2}\right)}{(i j)^{\beta-3}}, \cdots, \frac{\exp \left(i j \phi_{p \beta+\beta-2}^{\beta-2}\right)}{(i j)}, \exp \left(i j \theta_{p \beta+\beta-1}\right), \\
& \left.\quad \exp \left[i j \theta_{(p+1) \beta}\right]\right\},, p=0,1, \cdots, N-1 .
\end{aligned}
$$

Now consider the remaining integrals in (8); they are outside the product over $\gamma$ [the first two brackets (counting from the left)] and can be written

$$
\begin{aligned}
& \int_{\theta_{N \beta-2 \pi}}^{\theta_{\beta}} d \theta_{\beta-1} \int_{\theta_{\beta}}^{\theta_{2 \beta}} d \theta_{2 \beta-1} \int_{\theta_{2 \beta}}^{\theta_{3 \beta}} d \theta_{3 \beta-1} \ldots \int_{\theta_{(N-1) \beta}}^{\theta_{N \beta}} d \theta_{N \beta-1} \\
& \quad \times \prod_{\alpha=1}^{\beta-2}\left(\int_{\theta_{N \beta}-2 \pi}^{\theta_{\beta}} d \phi_{\alpha}^{\beta-2} \int_{\theta_{\beta}}^{\theta_{2 \beta}} d \phi_{\beta+\alpha}^{\beta-2} \int_{\theta_{2 \beta}}^{\theta_{3 \beta}} d \phi_{2 \beta+\alpha}^{\beta-2}\right. \\
& \left.\quad \times \ldots \int_{(N-1) \beta}^{\theta_{N \beta}} d \phi_{(N-1) \beta+\alpha}^{\beta-2}\right) .
\end{aligned}
$$

They operate on the determinant on the right-hand side of (21); the result is evident, and (21) is transformed into

$$
\begin{aligned}
& ( \pm 2)^{(\beta-1)(\beta-2) / 2} \operatorname{det}\left\{\frac{\exp \left(i j \theta_{\beta}\right)-\exp \left[i j\left(\theta_{N \beta}-2 \pi\right)\right]}{(i j)^{\beta-1}},\right. \\
& \times \frac{\exp \left(i j \theta_{\beta}\right)-\exp \left[i j\left(\theta_{N \beta}-2 \pi\right)\right]}{(i j)^{\beta-2}} \\
& \times \cdots, \frac{\exp \left(i j \theta_{\beta}\right)-\exp \left[i j\left(\theta_{N \beta}-2 \pi\right)\right]}{(i j)}, \\
& \left.\quad \times \exp \left(i j \theta_{\beta}\right), \cdots\right\}
\end{aligned}
$$

Here we have written only the first $\beta$ columns of the determinant. The effect on the other (groups of $\beta$ ) columns is exactly the same, except that $\theta_{\beta}$ is replaced by $\theta_{2 \beta}$ (columns $\beta+1$ through $2 \beta$ ), by $\theta_{3 \beta}$ (columns $2 \beta+1$ through $3 \beta), \cdots, \theta_{N \beta}$ [columns $(N-1) \beta+1$ through $N \beta$. If we let $p=\beta, 2 \beta, \cdots, N \beta$, we can add columns, and rewrite (23) so as to obtain

$$
\begin{gathered}
\operatorname{det}\left[\operatorname { e x p } ( i j \theta _ { s } ] \rightarrow ( \pm 2 ) ^ { ( \beta - 1 ) ( \beta - 2 ) / 2 } 2 ^ { \beta - 1 } \operatorname { d e t } \left[\frac{\exp \left(i j \theta_{p}\right)}{(i j)^{\beta-1}},\right.\right. \\
\left.\frac{\exp \left(i j \theta_{p}\right)}{(i j)^{\beta \circ 2}}, \cdots, \frac{\exp \left(i j \theta_{p}\right)}{(i j)}, \exp \left(i j \theta_{p}\right)\right] .
\end{gathered}
$$

Now multiply (24) by $K$ as defined in (9). This in effect multiplies the first row on the right-hand side of (24) by $\left(i j_{1}\right)^{\beta-1}$, the second row by $\left(i j_{2}\right)^{\beta-1}, \ldots$, the $\beta N$ th row by $\left(i j_{B N}\right)^{\beta-1}$. That is,

$$
\begin{aligned}
K \operatorname{det}\left[\exp \left(i j \theta_{s}\right)\right] \rightarrow( \pm 2)^{(\beta-1)(\beta-2) / 2} 2^{\beta-1} \operatorname{det}\left[\exp \left(i j \theta_{p}\right)\right. \\
\left.\quad \times(i j) \exp \left(i j \theta_{p}\right), \cdots,(i j)^{\beta-2} \exp \left(i j \theta_{p}\right),(i j)^{\beta-1} \exp \left(i j \theta_{p}\right)\right] \\
=( \pm 2)^{(\beta-1)(\beta-2) / 2} 2^{\beta-1}(i)^{N \beta(\beta-1) / 2} \operatorname{det}\left[\exp \left(i j \theta_{p}\right),\right. \\
\left.\quad \times j \exp \left(i j \theta_{p}\right), \cdots, j^{\beta-2} \exp \left(i j \theta_{p}\right), j^{\beta-1} \exp \left(i j \theta_{p}\right)\right] .
\end{aligned}
$$

This concludes the proof. The integrals on the lefthand side of $(8)$, operating on $\operatorname{det}\left[\exp \left(i j \theta_{s}\right)\right]$, produce the confluent alternant in (25), which is identical to the form (18) for $P_{N \prime \beta}{ }^{2}$. The remaining constant factors in (8) come from the definitions (13) and (14) of $P_{\beta N^{\prime} 1}$ and of $P_{N^{\prime} \beta^{2}}$ as determinants.

Alternative forms of the theorem, with different limits of integration, can easily be stated, but as indicated above, the form in (8) seems most natural. The first set of integrals in (8) [in the first square brackets in 
the first line of (8)], in their present form, reduce exactly to (7) in the case $\beta=2$ 。 The other integrals largely involve dummy variables as limits of integration, and changes in these may be rather arbitrary.

Finally, we note that $P_{N^{\prime} \beta^{2}}$ might be interpreted as the eigenvalue density for an ensemble of unitary matrices with $\beta^{2}$ independent components in each off-diagonal matrix element.

The application of (8) can be seen most easily in a simple example. Let $\beta N=6, \beta=3, N=2$. Then (8) becomes

$$
\begin{gathered}
\int_{\theta_{6}-2 r}^{\theta_{3}} d \theta_{2} \int_{\theta_{3}}^{\theta_{6}} d \theta_{5} \int_{\theta_{6}-2 \pi}^{\theta_{3}} d \phi_{1}^{1} \int_{\theta_{3}}^{\theta_{6}} d \phi_{4}^{1} \int_{\phi_{1}^{1}}^{\phi_{4}^{1}} d \theta_{1} \int_{\phi_{4}^{1}}^{\phi_{1}^{1+2 \pi}} d \theta_{4} P_{6^{\prime} 1}\left(\theta_{1}, \cdots, \theta_{6}\right) \\
=\left(\frac{2^{6} 3 !}{6 !}\right)^{4} \frac{C_{6^{\prime} 1}}{C_{2^{\prime} 9}} 2^{5} P_{2^{\prime} 9}\left(\theta_{3}, \theta_{6}\right) .
\end{gathered}
$$

The integrals in (26) can easily be performed in succession from right to left.

The statistical meaning of (26) can be seen in Fig. 1 . Two eigenvalues, $\theta_{1}$ and $\theta_{4}$, are chosen by the first two integrations, and allowed to move within the indicated interval; this destroys their ordering with respect to the other eigenvalues, but preserves it with respect to each other.

The third and fourth integrations fix $\phi_{4}^{1}$ and $\phi_{1}^{1}$ in the intervals $\left[\theta_{6}, \theta_{3}\right]$ and $\left[\theta_{3}, \theta_{6}\right]$, respectively, but without saying anything about the relation of $\phi_{4}^{1}$ to $\theta_{4}$ or $\theta_{5}$, or about the relation of $\phi_{1}^{l}$ to $\theta_{1}$ or $\theta_{2}$.

The last two integrations destroy the orderings of $\theta_{2}$ with respect to $\theta_{2}$ and $\theta_{3}$, and of $\theta_{5}$ with respect to $\theta_{4}$ and $\theta_{6}: \theta_{2}$ and $\theta_{5}$ are integrated out, just as the odd variables are in Theorem (7).

Note that allowing $N=2,4,6, \cdots$ ( $\beta N$ even) would make no significant change in (26). This is clear from Fig. 1, which for $N>2$ would simply be repeated horizontally.

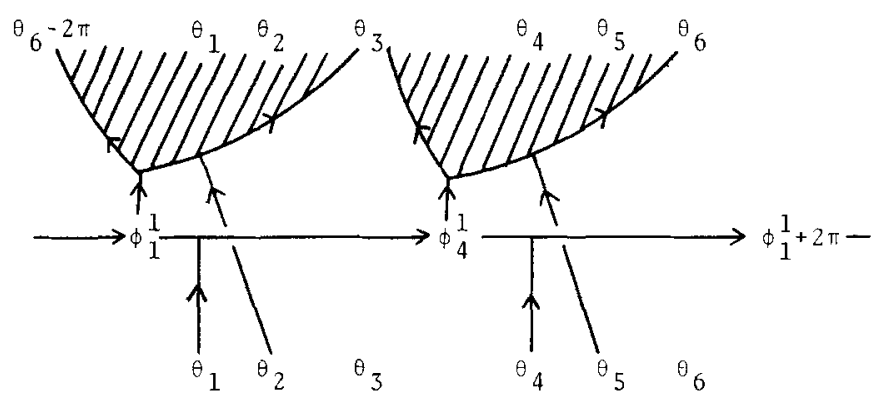

FIG. 1 .

From (26), the first integrations are performed over $\theta_{1}$ and $\theta_{4}$, in the intervals $\left[\phi_{1}^{1}, \phi_{4}^{1}\right]$ and $\left[\phi_{4}^{1}, \phi_{1}^{1}+2 \pi\right]$, respectively; the second set of integrations is over $\phi_{1}^{1}$ and $\phi_{4}^{1}$, in the intervals $\left[\theta_{6}-2 \pi, \theta_{3}\right]$ and $\left[\theta_{3}, \theta_{6}\right]$, respectively. The final set of integrations, which may in fact be performed at any time, is over $\theta_{2}$ and $\theta_{5}$, in the respective intervals $\left[\theta_{6}-2 \pi, \theta_{3}\right]$ and $\left[\theta_{3}, \theta_{6}\right]$. With the final integrations, the variables $\theta_{1}, \vartheta_{2}, \theta_{4}$ and $\theta_{5}$ have been eliminated.

If we were to allow $N=4,6,8, \cdots$, the diagram in $\mathrm{Fig}$. 1 would appear $2,3,4, \cdots$, times.

\section{ACKNOWLEDGMENT}

The author thanks Professor Alfred C. T. Wu for suggesting the problem, and for many useful discussions.

${ }^{1}$ F. J. Dyson, J. Math. Phys, 3, 140, 157, 166 (1962); F. J. Dyson and M. L. Mehta, J. Math. Phys. 4, 701 (1963).

${ }^{2}$ M. L. Mehta and F. J. Dyson, J. Math. Phys. 4, 713 (1963); M. L. Mehta, Random Matrices (Academic, New York, 1967)。 ${ }^{3}$ F. J. Dyson, J. Math. Phys. 3, 166 (1962).

${ }^{4} \mathrm{~J}$. Gunson, J. Math. Phys. 3, 752 (1962).

${ }^{5}$ A.C. Aitken, Determinants and Matrices (Interscience, New York, 1951).

${ }^{6} \mathrm{C} . \mathrm{E}$. Porter, Ed., Statistical Theories of Spectra: Fluctuations (Academic, New York, 1965), p. 62.

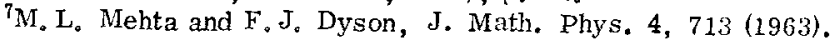

